\title{
Systematic Assessment of Surfactants for Matrix-Assisted Laser Desorption/ Ionization Mass Spectrometry Imaging
}

Bijay Banstola, Eulalie T. Grodner, ${ }^{\dagger}$ Fan Cao, Fabrizio Donnarumma, and Kermit K. Murray* 


\section{Abstract}

A systematic method for evaluation of MALDI profiling and imaging was developed and applied to the use of three surfactants, sodium dodecyl sulfate (SDS), Triton X-100, and Tween 20, on rat brain tissue. For profiling studies, mass spectra were acquired from regular arrays of spots with manually deposited surfactant and matrix. The studies recorded the total number of peaks in the mass spectra from 2 to $20 \mathrm{kDa}$ and compared the number of peaks and peak intensities with and without surfactant. It was found that SDS decreases the total number of peaks at all concentrations but does lead to an increase in the number of peaks below $5 \mathrm{kDa}$. Triton X-100 at $0.05 \%$ concentration yielded the highest number of peaks and highest number of new peaks, with the best results above $5 \mathrm{kDa}$. Correlation of the increase in signal with the estimated hydrophobicity suggests that Triton X-100 improves mass spectrometry quality through an increase in the intensity of hydrophobic protein peaks. Tween 20 provided good performance at $0.05 \%$ concentration across all mass ranges. For imaging studies, multiple images were obtained and the integrated intensity ratio for images obtained with and without surfactant was compared for 10 selected peaks. It was found that SDS tends to degrade imaging performance whereas Triton X-100 and Tween 20 improved performance compared to no surfactant, especially above $7 \mathrm{kDa}$. 


\section{Introduction}

Since its introduction nearly two decades ago [1], matrix-assisted laser desorption ionization imaging mass spectrometry (MALDI-IMS) has been used for identification and determination of the spatial distribution of proteins, peptides, lipids, metabolites, and other biomolecules in tissue [2-10]. For MALDI-IMS, tissue is cut using a microtome into $10 \mu \mathrm{m}$ thick sections that are mounted on a conductive microscope slide. A matrix solution is added either by droplet or spray deposition and the slide is mounted on a holder before being loaded into the mass spectrometer for analysis. Mass spectra are acquired sequentially at regular spatial intervals and the signal intensity for an ion of a particular mass is used to generate a heat map that indicates the molecule's location and concentration within the section. The lateral resolution is limited by the laser beam diameter, the raster step size, and the diffusion of the biomolecules due to matrix addition [11], The mass range is determined by mass analyzer and detector [12] as well as the method of sample preparation [13]. Digestion of proteins directly on tissue [14, 15] or after extraction from it [16-18] can be used to extend the effective mass range and aid in compound identification.

One of the most important factors affecting MALDI MSI performance is sample preparation and, in particular, the addition of the matrix to the tissue sample $[19,20]$. The matrix solution must be efficient at extracting biomolecules from the tissue for co-crystallization with the matrix while at the same time maintaining the analyte localization within the tissue, which is critical for good imaging resolution. Deposition of matrix droplets allows for efficient material extraction but limited lateral resolution and is often used in mass spectrometry profiling of selected tissue regions in what is known as profiling mode [21]. Spray deposition produces an even coating of 
matrix and is used for imaging mode at higher lateral resolution [22]. However, over wetting of the surface can lead to analyte delocalization.

Surfactants have been used in MALDI sample preparation to increase the signal intensity and number of peaks observed for hydrophobic biomolecules such as lipids and membrane proteins [23-27] and can improve the performance of MALDI mass spectrometry for complex mixtures of biomolecules. For example, when used with cell lysates, the mass range of MALDI analysis of cells was extended to $150 \mathrm{kDa}$ and the spectral quality markedly improved [28]. The addition of surfactants to whole cell bacteria analysis leads to new peaks observed in the mass range from 2 to $80 \mathrm{kDa}$ [29]. Surfactants can improve the performance of MALDI imaging as well. The nonionic surfactant Triton X-100 at a concentration of $0.5 \%$ was found to increase the number of peaks observed from 25 to $50 \mathrm{kDa}$ in MALDI imaging of rat and mouse kidney, heart, lung and brain tissue [30, 31]. Surfactants can also be used in conjunction with in situ trypsin digestion to aid protein identification $[32,33]$.

Despite the broad use of surfactants in MALDI and MALDI MSI, there have been limited systematic evaluations of surfactant performance. In the study described below, we have developed a method for systematic assessment of MALDI-MSI and have applied it to the use of three surfactants: the anionic surfactant sodium dodecyl sulfate (SDS), the nonionic polyethylene oxide chain surfactant Triton X-100, and the nonionic polysorbate surfactant Tween 20 . The surfactants were added to rat brain tissue sections by droplet deposition for profile mode analysis and pneumatic spray deposition for imaging mode from solutions containing sinapic acid matrix. The optimum concentration was determined and the relative performance of the surfactants was assessed based on the mass spectral peak count and peak intensity in different mass ranges and 
image quality. The performance improvement was correlated with the hydrophobicity index of identified proteins.

\section{Experimental}

Tissue samples were obtained from 4-6 week old breeding rats at the LSU School of Veterinary Medicine Division of Laboratory Animal Medicine (DLAM). The animals were sacrificed by $\mathrm{CO}_{2}$ (5 psi) exposure and brain samples were collected, washed in $50 \mathrm{mM}$ ammonium bicarbonate buffer for 30 seconds, and frozen in dry ice. Frozen samples were stored at $-80{ }^{\circ} \mathrm{C}$. Thin sections were prepared with a Leica CM1850 cryostat (Leica Microsystems, Wetzlar, Germany) directly from the frozen tissue. The tissue was fixed on a cryostat support with optimal cutting temperature compound (OCT), avoiding contact of the OCT solution with the exposed side of the tissue. Coronal or horizontal rat brain sections were cut at a thickness of $10 \mu \mathrm{m}$, thaw-mounted on indium tin oxide (ITO) coated microscope slides (Bruker Daltonics, Billerica, Massachusetts), and stored at $-80{ }^{\circ} \mathrm{C}$ until use. Horizontal sections were used for MALDI profiling whereas coronal sections were used for the imaging experiments. The tissue sections were vacuum dried for 10 min and washed with $70 \%$ ethanol and $95 \%$ ethanol for 30 seconds each followed by a chloroform wash for 15 seconds. The sections were vacuum dried for 10 min before matrix application.

For tissue profiling experiments, matrix was applied to tissue sections by manual spotting for profiling mode and by spraying for tissue imaging. For tissue profiling, a $3 \times 3$ array of spots with $2 \mathrm{~mm}$ spacing was selected on six consecutive tissue sections. Alignment of the spots from section to section was achieved by visual inspection. The matrix solution comprised $10 \mathrm{mg} / \mathrm{ml}$ of sinapic acid in a 2:1 (v/v) mixture methanol and $0.1 \%$ TFA and surfactant in ultrapure water. TFA was added to the mix to a final concentration of $0.1 \%$. A $200 \mathrm{~nL}$ volume of this solution 
was deposited on each spot and allowed to dry. This process was repeated five times for each spot. For each of the spots in the array, ten individual mass spectra were obtained summed.

Data analysis of profiling mode spectra was conducted using FlexAnalysis 3.0 software (Bruker). Spectra were baseline subtracted and smoothed using the Savitsky-Golay smoothing filter set at 10 Da width over three cycles. Identification was performed in centroid mode and peaks with signal-to-noise below 3 were not considered. MALDI images were processed using FlexImaging (Bruker) using the same peak detection method described above. Images were produced by integration of signals in a window of $0.2 \%$ of the $m / z$ of a detected peak.

For tissue imaging experiments, the matrix was spray deposited for tissue imaging using a pneumatic nebulizer that was constructed using a $200 \mu \mathrm{L}$ micropipette tip, a 0.25 in. stainless steel compression fitting (Swagelok, Solon Ohio), and a $75 \mu \mathrm{m}$ I.D. and $360 \mu \mathrm{m}$ O.D. silica capillary. The flow rate of the matrix solution was $100 \mu \mathrm{L} / \mathrm{min}$ and the nitrogen gas pressure was 10 PSI. The matrix was sprayed at a distance of $8 \mathrm{~cm}$ from the tissue and 14 spray cycles of $20 \mathrm{~s}$ each were used. The matrix was allowed to dry between each spray cycle.

MALDI mass spectra were recorded in positive ion linear mode with a tandem time-of-flight mass spectrometer (UltrafleXtreme, Bruker, Bremen, Germany). The instrument is equipped with a $355 \mathrm{~nm} \mathrm{Nd}$ :YAG laser operating at a repetition rate of $1 \mathrm{kHz}$. For profiling mode data acquisition, the laser was set to randomly irradiate an area of $0.05 \mathrm{~mm}^{2}$ around the selected position. A total of 100 laser shots were employed and ten individual mass spectra were recorded and summed to obtain the final spectrum of each spot. For imaging experiments, mass spectra were acquired over ca. $100 \mathrm{~mm}^{2}$ regions of tissue sections using a step size of $150 \mu \mathrm{m}$ and 500 laser shots per spectrum. After data acquisition, images were reconstructed using FlexImaging software (Bruker). A mass range of 2000-20,000 m/z was used for all experiments. 
The reagents chloroform, trifluoroacetic acid (TFA), ethanol, and methanol were purchased from Thermo Scientific (Waltham, Maryland). Sinapic acid (SA), sulfonyl dodecyl sulfate (SDS), and Triton X-100 and ammonium bicarbonate (ABC) were purchased from SigmaAldrich (St. Louis, Missouri). Tween 20 was purchased from Amresco (Solon, Ohio). Ultrapure water $(18 \mathrm{M} \Omega \mathrm{cm})$ was produced in-house.

\section{Results and Discussion}

Initial experiments were conducted in profiling mode to ascertain the optimum concentration and identify a set of peaks useful for quantifying the relative performance of the different surfactants. Mass spectra were obtained from horizontal sections with $1 \mu \mathrm{L}$ of matrix solution containing surfactants ranging from $0.001 \%$ to $0.50 \%$ concentration manually spotted on the tissue. A total of three mass spectra at each concentration were used to quantify the number of peaks in the range between 2000 and 20,000 m/z. Surfactant concentration above $0.1 \%$ resulted in a lower number of peaks (approximately one-half of that observed at lower concentrations) whereas surfactant concentration below that value resulted in a higher number of peaks for all surfactants. For the experiments described below, surfactant concentrations $0.075 \%$ and below were used.

Profiling mode experiments were conducted using surfactant concentrations of $0 \%, 0.001 \%$, $0.025 \%, 0.050 \%$, and $0.075 \%$. Five consecutive horizontal tissue sections were prepared as described above, each with a $3 \times 3$ array of spots. Three spots on each section were used for the three surfactants: Section 1 had no surfactant, Section 2 had 0.075\% surfactant, Section 3 had $0.050 \%$, Section 4 had $0.025 \%$, and Section 5 had $0.001 \%$. Figure 1 shows mass spectra obtained with surfactant compared to the same spot location with no surfactant. Figure 1a shows SDS at a 
surfactant concentration of $0.025 \%$, Figure $2 \mathrm{~b}$ shows Triton $\mathrm{X}-100$ at $0.050 \%$, and Figure $3 \mathrm{c}$ shows Tween 20 at $0.050 \%$, the optimum concentration for each surfactant. The addition of SDS led to a lower mass spectrum quality compared to the other surfactants. Triton X-100 produced substantial changes in spectrum intensity for many of the peaks; in addition, new peaks were detected. The mass spectrum with Tween 20 closely resembles the solvent-free mass spectrum although there are measurable peak intensity differences. The proteins that were identified in the mass spectra are pro-neuropeptide Y (MW 3464) [34], thymosin $\beta 4$ (MW 4963) [34], COX8B (MW 4990) [35], COX8A (MW 5455) [35], PEP 19 (MW 6718) [34], neurogranin (MW 7531) [36], ubiquitin (MW 8566) [34], COX6B (MW 9979) [34], myelin basic protein (MW 14,127) [37], and rMAL (MW 16,331) [38].

To quantify the changes in the mass spectra upon addition of surfactant, the total number of peaks between 2000 and $20,000 \mathrm{~m} / \mathrm{z}$ for surfactants SDS, Triton X-100 and Tween 20 was measured. Figure 2 displays the results from the evaluation conducted on the spectra from the profiling mode experiments. SDS reduced the number of peaks detected: at $0 \%$ concentration, 51 peaks were observed, but this number was roughly halved with a surfactant concentration of $0.050 \%$ and above (Figure 2a). For Triton X-100 and Tween 20, the number of peaks observed was comparable at all concentration ranges.

Although in most cases the mass spectra had comparable numbers of peaks at the different surfactant concentrations, these peaks were not the same. The addition of surfactant often led to the loss of peaks compared to the comparable surfactant-free mass spectrum as well as the appearance of new peaks in the surfactant mass spectra. The number of peaks detected in the surfactant mass spectra compared to the corresponding surfactant-free spectra ("new" peaks) is shown in Figure 2b. For SDS, almost half of the total peaks detected at surfactant concentrations 
higher than $0.001 \%$ are new peaks that are unique to these mass spectra, whereas for Triton $\mathrm{X}$ 100 and Tween 20, the number of new peaks observed at the same surfactant concentrations is about one-third.

It was found that the appearance of new peaks as well as an increase in the intensity of existing peaks is dependent on the $\mathrm{m} / \mathrm{z}$ region selected. Figure 3 shows the number of peaks that are new or have a greater intensity in the presence of surfactant in different mass ranges. A peak was considered to have a greater intensity if its height was greater than that of the peak in the corresponding surfactant-free mass spectrum plus one standard deviation. It can be seen that most of the new and enhanced peaks for SDS are between 2-5 kDa (assuming singly charged ions). Triton X-100 produces new and enhanced peaks above $5 \mathrm{kDa}$, particularly in the 5 to 10 $\mathrm{kDa}$ region. The new and enhanced peaks for Tween 20 are comparable in all mass ranges.

The addition of proteins of known concentration as internal standards was used to assess the effect of the surfactants on proteins of known concentration deposited on the tissue. If the addition of surfactant primarily affects extraction of proteins from the tissue and not other MALDI parameters such as co-crystallization, the intensity of a protein internal standard could be used for quantification in profiling and imaging experiments. Four consecutive tissue sections were spiked as described above using a matrix solution containing the protein standards insulin, cytochrome $\mathrm{C}$ and myoglobin. A total of 9 position were arrayed on each of the four sections. Three spots on each section were used for the three protein standards. The first section had no surfactant, the second had $0.025 \%$ SDS, the third had $0.050 \%$ Triton X-100, and the fourth had $0.050 \%$ Tween 20 . Figure $\mathrm{S} 1$ shows the average intensity for the $[\mathrm{M}+\mathrm{H}]^{+}$peak for each protein and the different surfactants. As can be seen from the figure, the effect of the surfactant depends on the protein standard used. For example, insulin has $46 \%$ of the no surfactant control signal 
with SDS and $2 \%$ with Triton X-100, whereas cytochrome $\mathrm{C}$ has $7 \%$ of the control signal with SDS and $205 \%$ with Triton X-100. Some of the results are similar to those obtained for the tissue-bound proteins in the profiling experiments. For example, SDS improved the signal for lower molecular weight proteins (Figure 3a) whereas Triton X-100, and to a lesser extent Tween 20 , performed better for higher molecular weight proteins (figure $3 b$ and $3 c$ ). This is consistent with SDS improving the insulin signal whereas Triton X-100 and Tween 20 improve the signal for the higher molecular weight cytochrome $\mathrm{c}$ and myoglobin. These results suggest that the surfactants influence the solubilization and matrix co-crystallization in addition to tissue extraction. Due to the fact that effect of the surfactant depends strongly on the protein standard used, internal standards were not used for the subsequent experiments.

The results shown in Figures 2 and 3 were used to identify the concentrations of surfactant suitable for the imaging experiments. For the three surfactants, concentrations of $0.025 \%$ and $0.050 \%$ give the best results in terms of number of peaks and new and enhanced peaks in the mass spectra. The SDS surfactant performs better at lower concentrations and thus a concentration of $0.025 \%$ was used for the imaging experiments and $0.050 \%$ was used for Triton X-100 and Tween 20.

Imaging experiments were done by spraying matrix mixture on $10 \mu \mathrm{m}$ rat brain sections using the pneumatic sprayer described in the Experimental section above. MALDI images constructed from selected $\mathrm{m} / \mathrm{z}$ values $(5009,6222,6646,6758,8565,8601,9975)$ corresponding to the centroids of several common intense peaks are shown in Figure 4. For each set of images two sections were mounted on a slide; one was sprayed with matrix solution containing surfactant and the other with a solution containing no surfactant. The spray solution contained 
0.025\% SDS (Column 1), 0.050\% Triton X-100 (Column 2), and 0.050\% Tween 20 (Column 3). Imaging experiments were repeated three times for each surfactant with comparable results.

The relative intensity of ten peaks was determined from triplicate sets of MALDI images by integrating the peak height and dividing by the image area. The ratios of the integrated intensities with surfactant to the integrated intensities without surfactant are shown in Figure 5. The SDS surfactant images have a lower integrated signal compared to surfactant-free, a result similar to that from the profiling experiments. Triton X-100 has a higher integrated intensity for most of the $\mathrm{m} / \mathrm{z}$ values, particularly for those in the $\mathrm{m} / \mathrm{z}$ range above 7500 , a result that follows the same trends seen in the profiling experiments. The Tween 20 intensities are largely unaffected by the surfactant with the exception of 9975 and $12,131 \mathrm{~m} / \mathrm{z}$, again a result similar to the profiling study.

Figure 6 shows pairs of images obtained from $\mathrm{m} / \mathrm{z} 6720,7573$ and 14135 for each surfactant; the left image for each pair was obtained without surfactant. The $\mathrm{m} / \mathrm{z} 6720$ image corresponds to the PEP 19 protein and the $\mathrm{m} / \mathrm{z}$ 14,135 to myelin basic protein. The composite image in the right column is an overlay of the three images for the three proteins.

Our general observations in both profiling mode and imaging mode indicate that Triton X100 had the best overall performance in terms of number of peaks, new peaks observed, and mass spectrum intensity, particularly in the mass range above $5 \mathrm{kDa}$. Our results are consistent with previous reports of Triton X-100 as the best performing surfactant for brain tissue [31]. Tween 20 had good performance across all of the mass ranges, consistent with previous reports $[28,31]$, although it should be noted that the relative performance of the surfactants depends on tissue type and the matrix deposition method [31]. We observed that the addition of SDS tended to degrade performance both in profiling as well as imaging mode. In prior studies, SDS has 
been shown to improve performance for liver and heart tissue [31] and relatively high concentration of SDS has been shown to improve MALDI performance [25]. The surfactant SDS has been used successfully in conjunction with careful sample preparation including vortexing to disrupt micelle formation [27]; however, degraded performance is often observed [39, 40]. Our observation that SDS provides relatively poor performance for profiling and imaging of brain tissue is consistent with these previous results.

The correlation between protein hydrophobicity and signal increase on surfactant addition can be assessed using an amino acid hydrophobicity scale. The hydrophobicity of ten proteins observed in profiling mode was estimated using a Kyte-Doolittle scale and a window size of 21 [41]. The percent increase in peak intensity for proteins in profiling mode mass spectra (Figure 2) for ten proteins was plotted against the hydrophobicity index of the proteins. The plots for the three surfactants are shown in supplementary Figure S2. The slope of the plot for Triton X-100 is 92.5 with an R-squared value of 0.8 , indicating that the addition of surfactant leads to improved signal for the more hydrophobic proteins. The correlation for Tween 20 is weaker with a slope of 0.21 and R-squared value of 0.3. For SDS, the slope is 1.8 with an R-squared value of 0.02 , suggesting no significant correlation. These observations suggest that the Triton X-100 consistently improves the performance of MALDI imaging by enhancing the signal observed for hydrophobic proteins, leading to more peaks and higher intensities.

The proposed mechanism for surfactant enhancement of MALDI imaging performance is that the surfactant prevents protein aggregation [28]. The surfactant forms smaller micelles containing the hydrophobic species with surfactant forming micelles that co-crystallize with the matrix as the carrier solvent evaporates. However, the micelle may also displace cytoskeletal and extracellular proteins that help maintain the tissue porosity, resulting in lower extraction 
efficiency [30]. The effect of the various surfactants on various tissue types leads to the different performance observed $[30,31]$. When combined with the dependence on surfactant composition, matrix, solvent and deposition method, these differences lead to an overall wide variation in performance of the surfactants.

\section{Conclusions}

We have developed a method for systematic evaluation of tissue profiling and imaging by MALDI and have applied the approach to the use of surfactants for improved signal intensity and detection of new peaks in the mass spectra from rat brain tissue analysis. For profiling, the total number of peaks observed and the number of new peaks detected at different surfactant concentrations up to $0.075 \%$ were recorded. For imaging, the relative intensities of selected peaks with and without surfactant were obtained. We found that the nonionic surfactant Triton $\mathrm{X}-100$ gave the best performance for both profiling and imaging and that this is due to higher signal from hydrophobic proteins. Triton X-100 was particularly useful for proteins above $5 \mathrm{kDa}$ in mass. Tween 20 had good performance across the mass range from 2 to $20 \mathrm{kDa}$. The anionic surfactant SDS tended to degrade performance under most conditions. Future studies will apply this approach to additional tissue types and sample preparation methods. In situ trypsin digestion will be used to aid protein identification and parallel LCMS/MS analysis using serial consecutive sections will allow further validation of the quantitative results obtained.

\section{Acknowledgments}

This work was supported by the National Science Foundation (Grant No. CHE-1152106).

The authors thank Dr. D. Baker (School of Veterinary Medicine, Louisiana State University, 
Baton Rouge, LA) for kindly providing rat brain samples. ETG thanks the Louisiana Biomedical Research Network for summer research support. 


\section{References}

[1] R.M. Caprioli, T.B. Farmer, J. Gile, Molecular imaging of biological samples: localization of peptides and proteins using MALDI-TOF MS, Anal. Chem., 69 (1997) 4751-4760.

[2] L. McDonnell, R.M. Heeren, Imaging mass spectrometry, Mass Spectrom. Rev., 26 (2007) 606-643.

[3] R.C. Murphy, J.A. Hankin, R.M. Barkley, Imaging of lipid species by MALDI mass spectrometry, J. Lipid Res., 50 (2009) S317-S322.

[4] S.S. Rubakhin, J.V. Sweedler, A mass spectrometry primer for mass spectrometry imaging., Methods Mol. Biol., 656 (2010) 21-49.

[5] P. Chaurand, Imaging mass spectrometry of thin tissue sections: A decade of collective efforts, J. Proteomics, 75 (2012) 4883-4892.

[6] J.L. Norris, R.M. Caprioli, Analysis of Tissue Specimens by Matrix-Assisted Laser Desorption/Ionization Imaging Mass Spectrometry in Biological and Clinical Research, Chem. Rev., 113 (2013) 2309-2342.

[7] M. Shariatgorji, A. Nilsson, R.J. Goodwin, P. Källback, N. Schintu, X. Zhang, A.R. Crossman, E. Bezard, P. Svenningsson, P.E. Andren, Direct targeted quantitative molecular imaging of neurotransmitters in brain tissue sections, Neuron, 84 (2014) 697-707.

[8] M.M. Gessel, J.L. Norris, R.M. Caprioli, MALDI imaging mass spectrometry: Spatial molecular analysis to enable a new age of discovery, J. Proteomics, (2014) 1-12.

[9] R.M.A. Heeren, Getting the picture: The coming of age of imaging MS, Int. J. Mass Spectrom. , (2014) 1-9.

[10] A. Bodzon-Kulakowska, P. Suder, Imaging mass spectrometry: Instrumentation, applications, and combination with other visualization techniques, Mass Spectrom. Rev., 35 (2015) 147-169.

[11] K.K. Murray, C.A. Seneviratne, S. Ghorai, High resolution laser mass spectrometry bioimaging, Methods, (2016) 1-9.

[12] A. van Remoortere, R.J.M. van Zeijl, N. van den Oever, J. Franck, R. Longuespée, M. Wisztorski, M. Salzet, A.M. Deelder, I. Fournier, L.A. McDonnell, MALDI imaging and profiling MS of higher mass proteins from tissue, J. Am. Soc. Mass Spectrom., 11 (2010) 19221929.

[13] V. Mainini, G. Bovo, C. Chinello, E. Gianazza, M. Grasso, G. Cattoretti, F. Magni, Detection of high molecular weight proteins by MALDI imaging mass spectrometry, Mol. Biosyst., 9 (2013) 1101-1107. 
[14] M.R. Groseclose, M. Andersson, W.M. Hardesty, R.M. Caprioli, Identification of proteins directly from tissue:in situ tryptic digestions coupled with imaging mass spectrometry, J. Mass Spectrom., 42 (2007) 254-262.

[15] B. Cillero-Pastor, R.M.A. Heeren, Matrix-assisted laser desorption ionization mass spectrometry imaging for peptide and protein analyses: a critical review of on-tissue digestion., J. Proteome Res., 13 (2014) 325-335.

[16] J.J. Nicklay, G. Harris, K. Schey, R.M. Caprioli, MALDI imaging and in situ identification of integral membrane proteins from rat brain tissue sections, Anal. Chem., (2013).

[17] J. Quanico, J. Franck, J.P. Gimeno, R. Sabbagh, M. Salzet, R. Day, I. Fournier, Parafilmassisted microdissection: a sampling method for mass spectrometry-based identification of differentially expressed prostate cancer protein biomarkers., Chem. Com., 51 (2015) 4564-4567.

[18] F. Donnarumma, K.K. Murray, Laser ablation sample transfer for localized LC-MS/MS proteomic analysis of tissue., J. Mass Spectrom., 51 (2016) 261-268.

[19] W. Bouschen, B. Spengler, Artifacts of MALDI sample preparation investigated by highresolution scanning microprobe matrix-assisted laser desorption/ionization (SMALDI) imaging mass spectrometry, Int. J. Mass Spectrom., (2007).

[20] P. Chaurand, Introducing specificity and sensitivity in imaging MS, Bioanalysis, 7 (2015) 2279-2281.

[21] P. Chaurand, J. Norris, D. Cornett, J. Mobley, R.M. Caprioli, New developments in profiling and imaging of proteins from tissue sections by MALDI mass spectrometry, J. Proteome Res., 5 (2006) 2889-2900.

[22] H.-Y.J. Wang, S.N. Jackson, J. Post, A.S. Woods, A Minimalist Approach to MALDI Imaging of Glycerophospholipids and Sphingolipids in Rat Brain Sections., Int. J. Mass Spectrom., 278 (2008) 143-149.

[23] B. Rosinke, K. Strupat, F. Hillenkamp, J. Rosenbusch, N. Dencher, U. Krüger, H.-J. Galla, Matrix-assisted laser desorption/ionization mass spectrometry (MALDI-MS) of membrane proteins and non-covalent complexes, J. Mass Spectrom., 30 (1995) 1462-1468.

[24] S.L. Cohen, B.T. Chait, Influence of matrix solution conditions on the MALDI-MS analysis of peptides and proteins, Anal. Chem., 68 (1996) 31-37.

[25] F.M.L. Amado, M.G. Santana-Marques, A.J. Ferrer-Correia, K.B. Tomer, Analysis of Peptide and Protein Samples Containing Surfactants by MALDI-MS, Anal. Chem., 69 (1997) 1102-1106.

[26] M. Cadene, B.T. Chait, A robust, detergent-friendly method for mass spectrometric analysis of integral membrane proteins., Anal. Chem., 72 (2000) 5655-5658. 

laser desorption/ionization mass spectrometry of hydrophobic and hydrophilic peptides, Anal. Chem., 72 (2000) 1169-1174.

[28] K.O. Börnsen, M.A.S. Gass, G.J.M. Bruin, J.H.M. von Adrichem, M.C. Biro, G.M. Kresbach, M. Ehrat, Influence of Solvents and Detergents on Matrix-assisted Laser Desorption/Ionization Mass Spectrometry Measurements of Proteins and Oligonucleotides, Rapid Commun. Mass Spectrom., 11 (1997) 603-609.

[29] M.A. Meetani, K.J. Voorhees, MALDI mass spectrometry analysis of high molecular weight proteins from whole bacterial cells: Pretreatment of samples with surfactants, J. Am. Soc. Mass Spectrom., 16 (2005) 1422-1426.

[30] B.D. Leinweber, G. Tsaprailis, T.J. Monks, S.S. Lau, Improved MALDI-TOF imaging yields increased protein signals at high molecular mass., J. Am. Soc. Mass Spectrom., 20 (2009) 89-95.

[31] V. Mainini, P.M. Angel, F. Magni, R.M. Caprioli, Detergent enhancement of on-tissue protein analysis by matrix-assisted laser desorption/ionization imaging mass spectrometry, Rapid Commun. Mass Spectrom., 25 (2011) 199-204.

[32] E. Patel, M.R. Clench, A. West, P.S. Marshall, N. Marshall, S. Francese, Alternative Surfactants for Improved Efficiency of In Situ Tryptic Proteolysis of Fingermarks, J. Am. Soc. Mass Spectrom., 26 (2015) 862-872.

[33] M.-C. Djidja, S. Francese, P.M. Loadman, C.W. Sutton, P. Scriven, E. Claude, M.F. Snel, J. Franck, M. Salzet, M.R. Clench, Detergent addition to tryptic digests and ion mobility separation prior to $\mathrm{MS} / \mathrm{MS}$ improves peptide yield and protein identification for in situproteomic investigation of frozen and formalin-fixed paraffin-embedded adenocarcinoma tissue sections, Proteomics, 9 (2009) 2750-2763.

[34] H. Ye, R. Mandal, A. Catherman, P.M. Thomas, N.L. Kelleher, C. Ikonomidou, L. Li, Topdown proteomics with mass spectrometry imaging: a pilot study towards discovery of biomarkers for neurodevelopmental disorders, PloS one, 9 (2014) e92831.

[35] P. Schindler, A. Vandorsselaer, A. Falick, Analysis of hydrophobic proteins and peptides by electrospray ionization mass spectrometry, Anal. Biochem., 213 (1993) 256-263.

[36] L.S. Eberlin, X. Liu, C.R. Ferreira, S. Santagata, N.Y. Agar, R.G. Cooks, Desorption electrospray ionization then MALDI mass spectrometry imaging of lipid and protein distributions in single tissue sections, Anal. Chem., 83 (2011) 8366-8371.

[37] A. Zavalin, J. Yang, K. Hayden, M. Vestal, R.M. Caprioli, Tissue protein imaging at $1 \mu \mathrm{m}$ laser spot diameter for high spatial resolution and high imaging speed using transmission geometry MALDI TOF MS, Anal. Bioanal. Chem., 407 (2015) 2337-2342. 
[38] N. Schaeren-Wiemers, D. Valenzuela, M. Frank, M. Schwab, Characterization of a rat gene, rMAL, encoding a protein with four hydrophobic domains in central and peripheral myelin, J. Neurosci., 15 (1995) 5753-5764.

[39] F. Xiang, R.C. Beavis, Growing protein-doped sinapic acid crystals for laser desorption: An alternative preparation method for difficult samples, Org. Mass Spectrom., 28 (1993) 1424-1429.

[40] S.M. Mandal, S. Dey, M. Mandal, S. Maria-Netob, O.L. Francob, Comparative analyses of different surfactants on matrix-assisted laser desorption/ionization mass spectrometry peptide analysis, Eur. J. Mass Spectrom., 16 (2010) 567-575.

[41] J. Kyte, R.F. Doolittle, A simple method for displaying the hydropathic character of a protein, J. Mol. Biol., 157 (1982) 105-132. 


\section{Figure Captions}

Figure 1. Profiling mode mass spectra without surfactant (upper) and with surfactant (lower) for a) SDS at $0.025 \%$, b) Triton X-100 at $0.050 \%$, and c) Tween-20 at $0.050 \%$.

Figure 2. Number of peaks observed in MALDI profiling between 2000 and 20,000 $\mathrm{m} / \mathrm{z}$ with different concentration of surfactant: a) total number of peaks and b) number of peaks in surfactant mass spectrum that do not appear in the surfactant-free mass spectrum.

Figure 3. Number of new peaks and higher intensity peaks found in MALDI profiling sorted by mass range: a) SDS; b) Triton 100; c) Tween 20.

Figure 4. Pairs of MALDI images of rat brain coronal sections obtained with surfactant (right) and without surfactant (left) for selected $\mathrm{m} / \mathrm{z}$ and surfactant.

Figure 5. Intensity ratio of ten selected peaks obtained from MALDI images with and without surfactant.

Figure 6. Pairs of images corresponding to 6720,7573 and $14135 \mathrm{~m} / \mathrm{z}$ for SDS, Triton X-100, and Tween 20 surfactants on rat brain coronal sections; the left image for each pair was obtained without surfactant. 
Figure 1
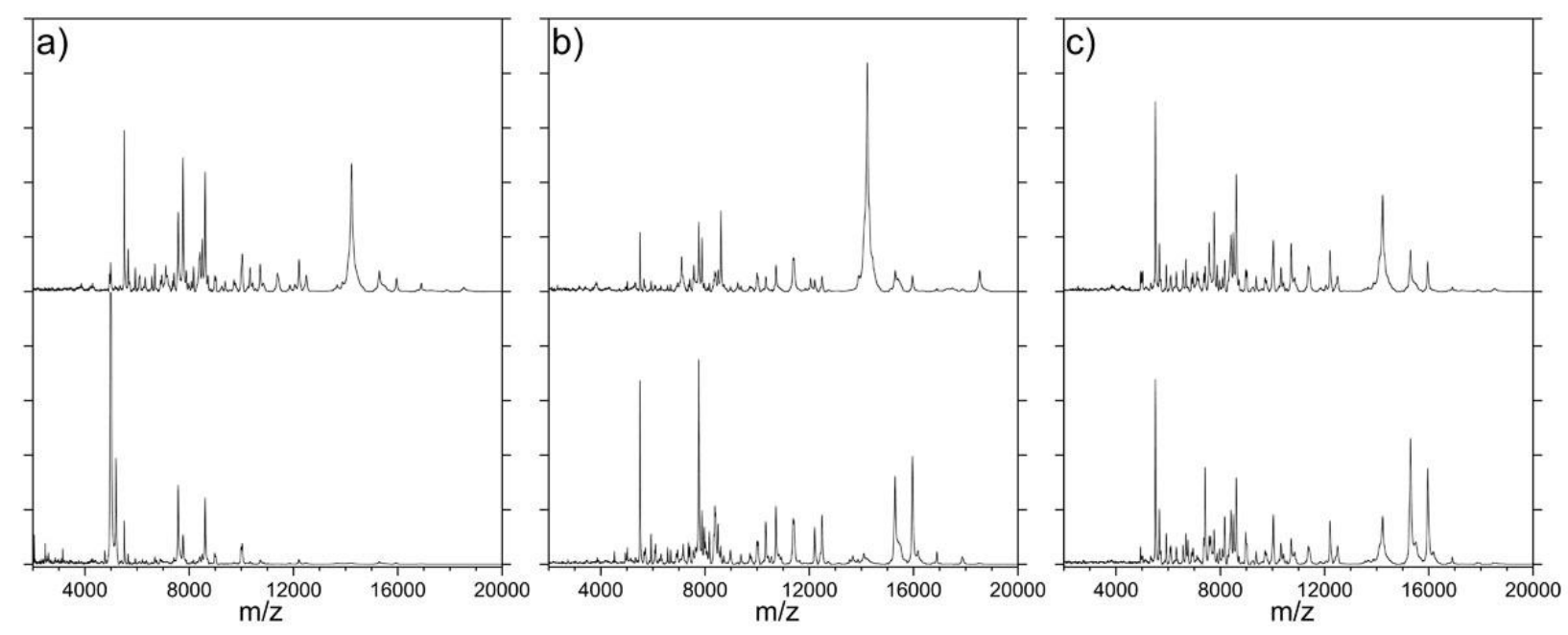

24

25

26

27

28

29

30

31

32

33

34

35

36

37

38

39

40

41

42

43

44

45

46

47

48

49

50

51

52

53

54

55

56

57

58

59

60

61

62 
Figure 2

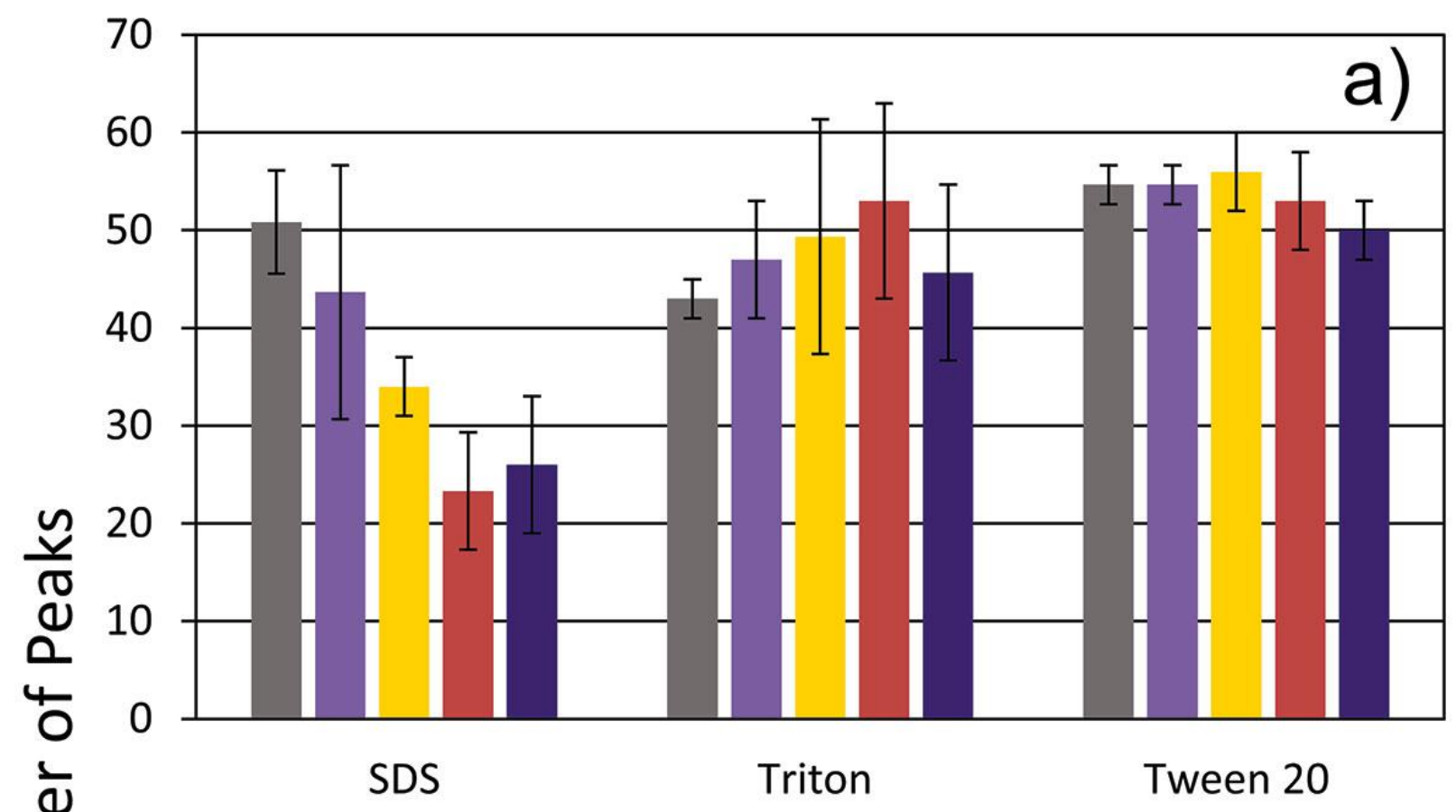


Figure 3

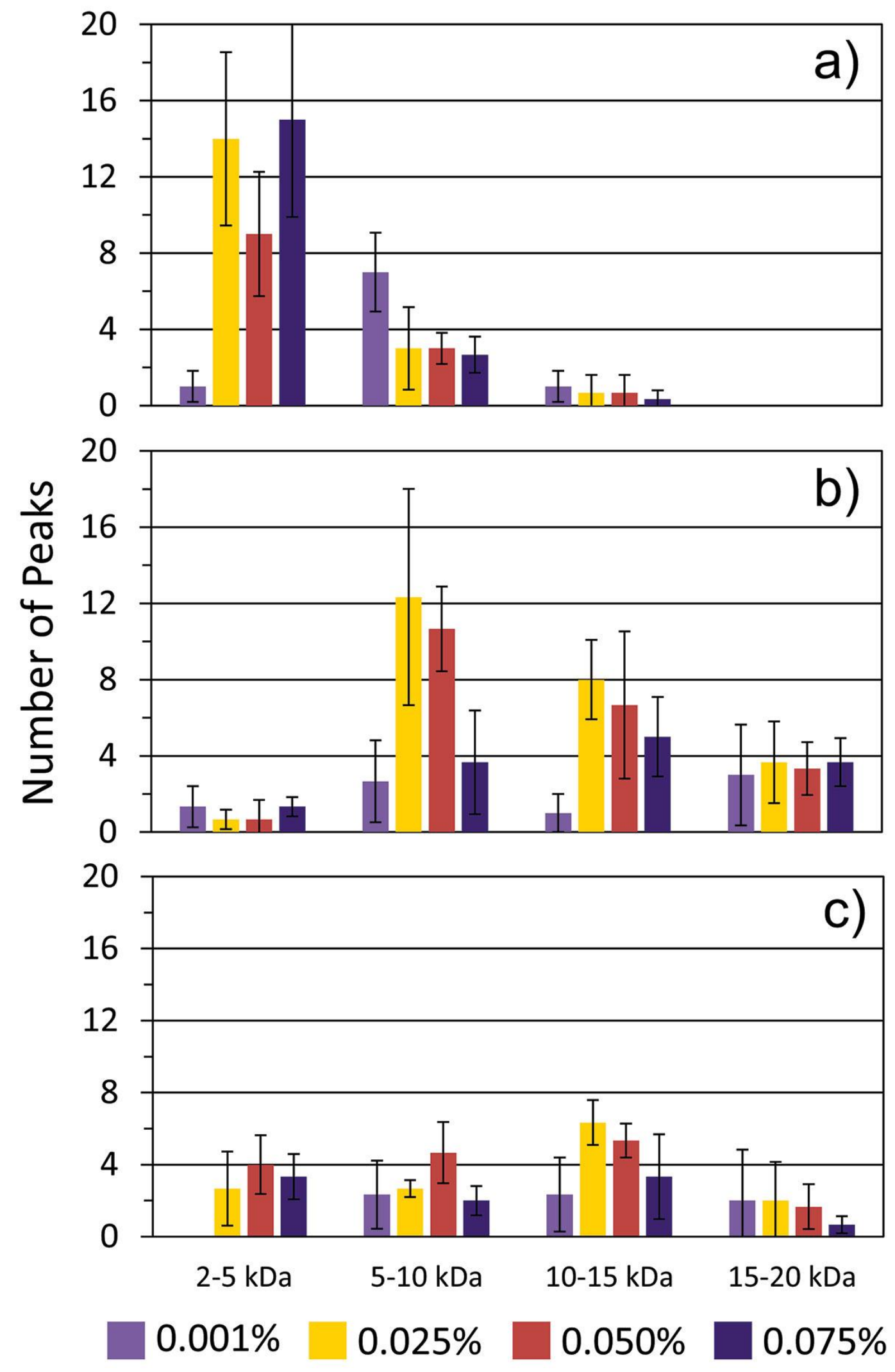




\section{Figure 4}

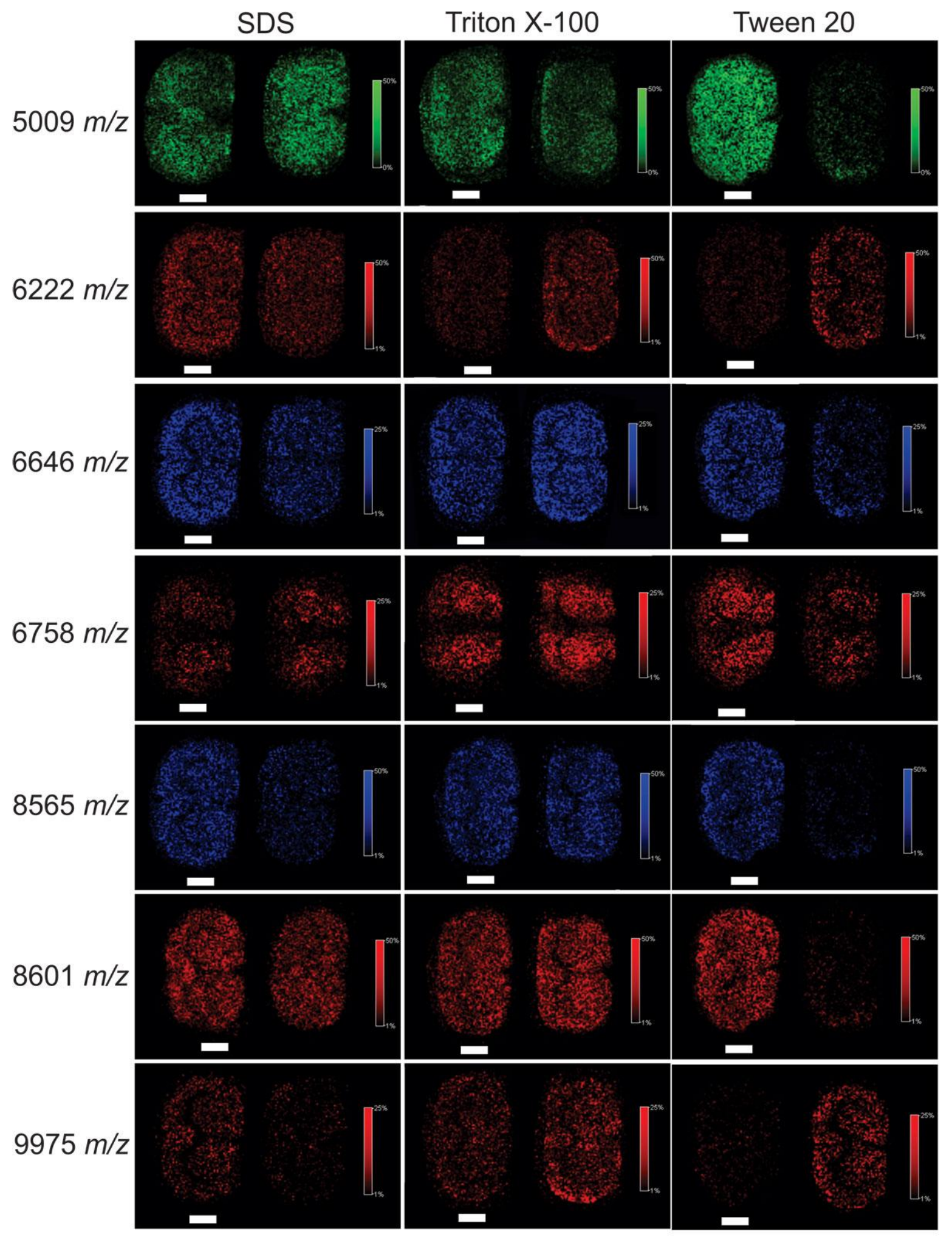




\section{Figure 5}
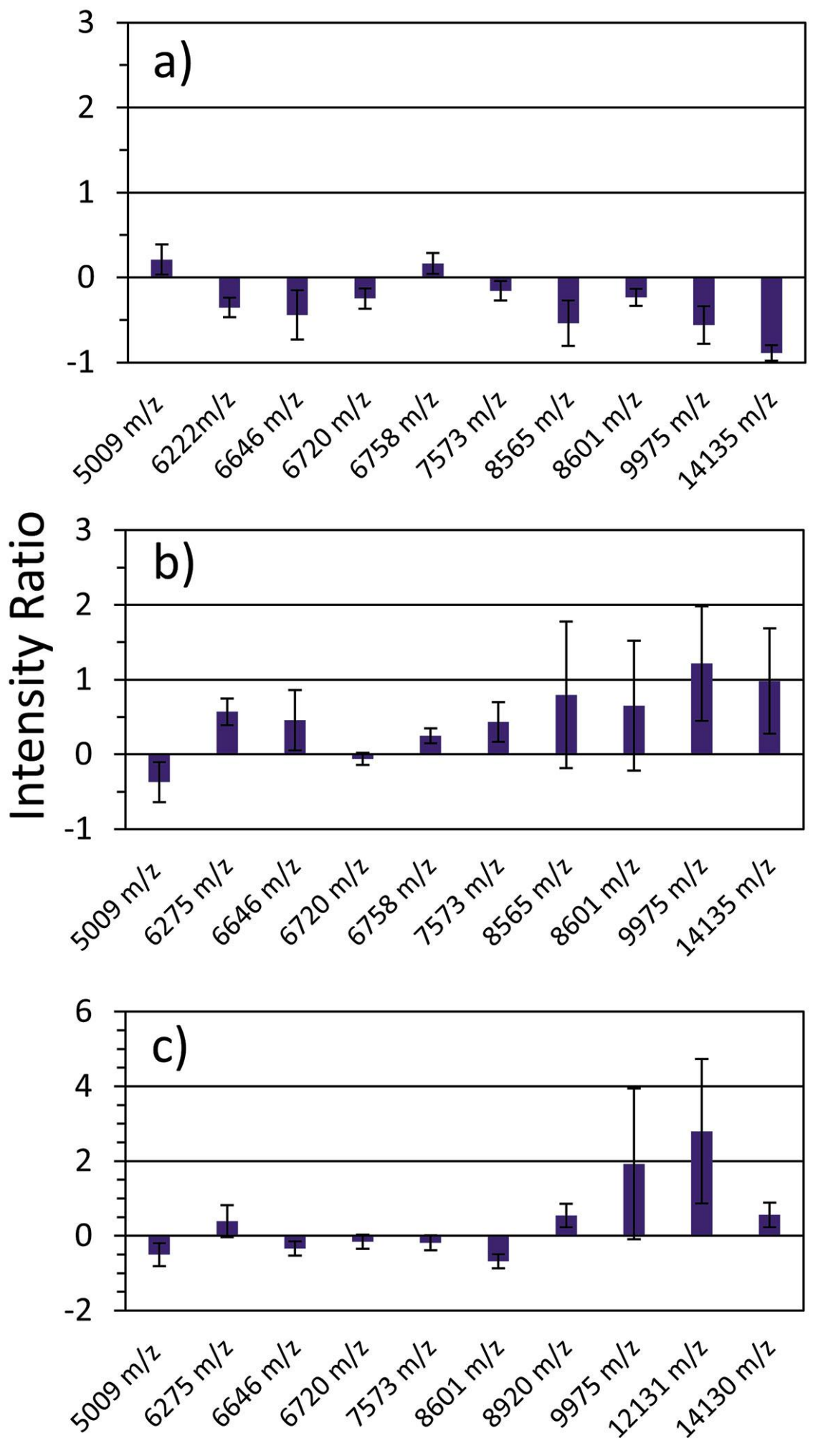
Figure 6

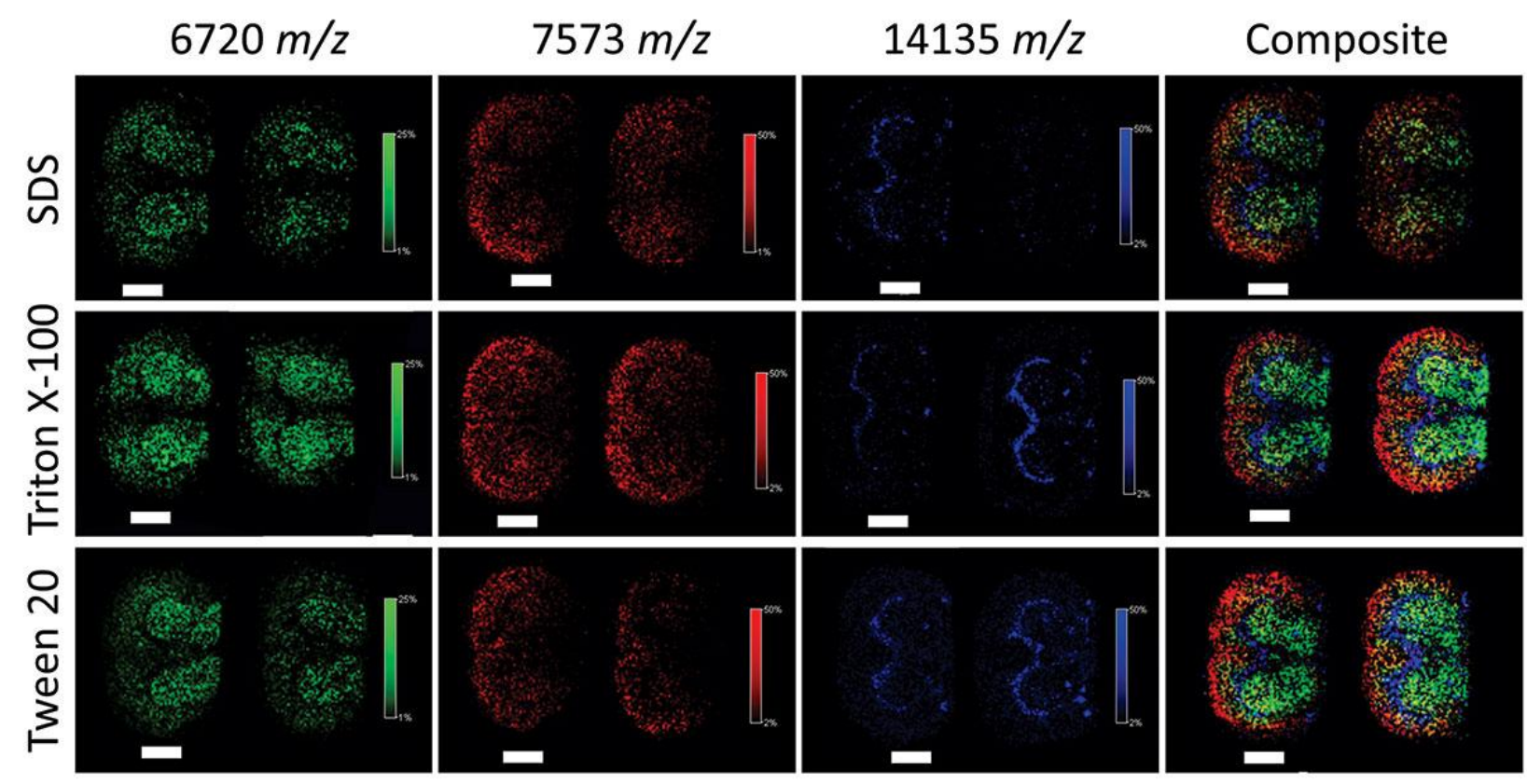

29

30

31

32

33

34

35

36

37

38

39

40

41

42

43

44

45

46

47

48

49

50

51

52

53

54

55

56

57

58

59

60

61

62

63 

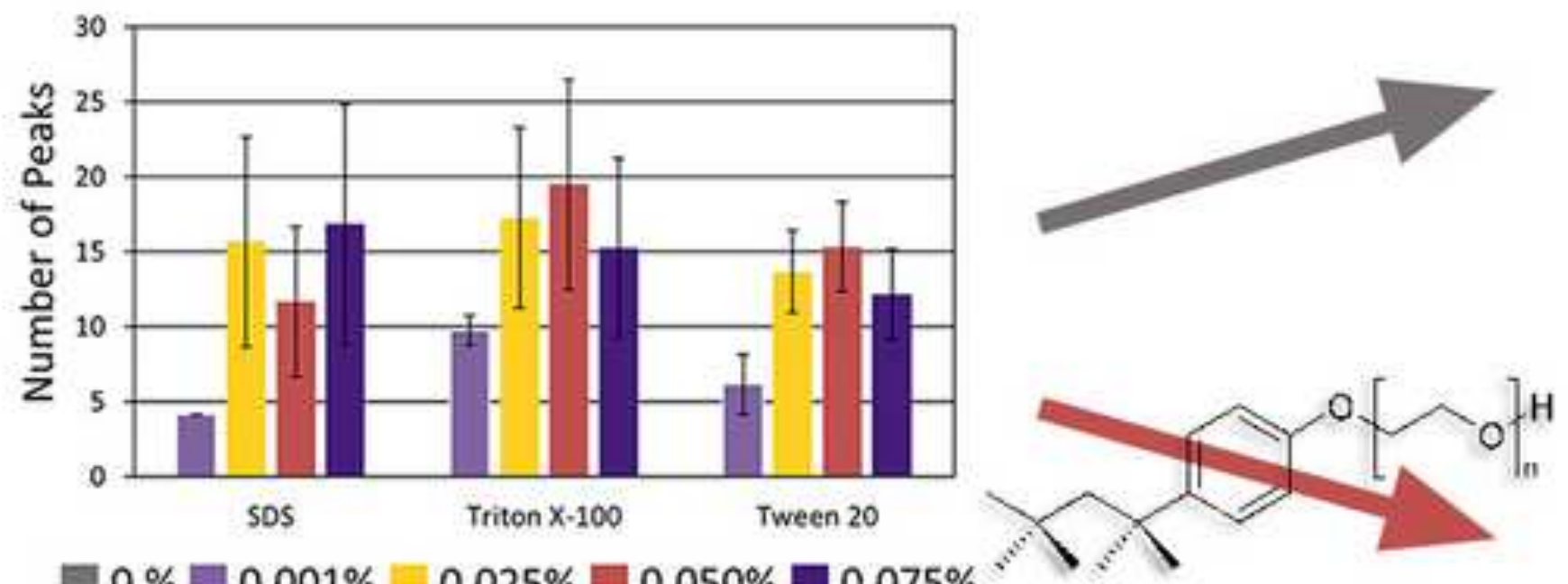

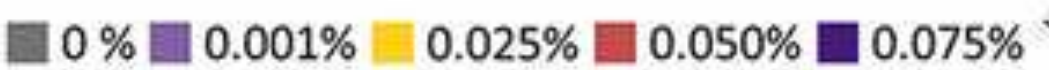

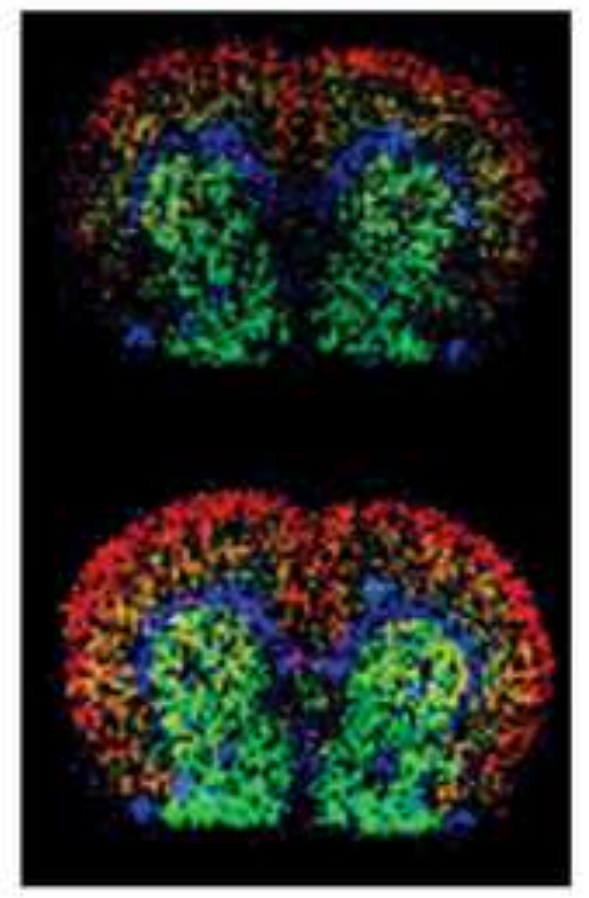

